\title{
What is the origin of dyspnoea in smokers without airway disease?
}

\author{
Enrico M. Clini $\mathbb{1}^{1}$, Bianca Beghé ${ }^{1}$ and Leonardo M. Fabbri $\mathbb{1}^{2}$
}

Affiliations: ${ }^{1}$ Dept of Medical and Surgical Sciences, University of Modena and Reggio Emilia, Modena, Italy. ${ }^{2}$ Dept of Medicine, Endocrinology, Geriatrics and Metabolism - Sant'Agostino Estense Hospital, University of Modena and Reggio Emilia, Modena, Italy.

Correspondence: Leonardo M. Fabbri, Dept of Medicine, Endocrinology, Geriatrics and Metabolism, Section of Metabolic Medicine, University of Modena and Reggio Emilia, Nuovo Ospedale Civile Sant'Agostino Estense, Via Giardini 1355, I-41126 Modena, Italy. E-mail: leonardo.fabbridunimore.it

-

@ERSpublications

Exertional dyspnoea is a debilitating symptom of smokers; understanding its mechanisms might allow better treatments http://ow.ly/sbQV3023L9A

Dyspnoea is a symptom that affects quality of life, exercise intolerance and prognosis in various chronic conditions (figure 1). As a consequence of dyspnoea, approximately half of all patients with chronic obstructive pulmonary disease (COPD) report a significant degree of disability, losing one or more functions in daily life [1]. Quantification of dyspnoea is therefore essential, and can be achieved through specific instruments (scales and/or questionnaires) that define the intensity rate and relief following pharmacological and nonpharmacological therapies. Several measures pertaining to domains of sensoryperceptual experience (what breathing feels like), affective distress or symptom burden/impact are used [2]. A modified Barthel Index (a scale to assess an individual's general disability) was recently validated to quantify the extent to which functional abilities are limited by dyspnoea in chronic respiratory conditions, including COPD [3]. However, complex physiological mechanisms that generate dyspnoea in single categories of chronic illness are poorly understood.

Recent, large population studies revealed patients with chronic symptoms, mainly chronic dyspnoea, and normal spirometry, i.e. without documented functional alteration of the respiratory system $[4,5]$. The last observational study in an adult population of smokers (SPIROMICS) demonstrated that symptomatic current or former smokers with normal pulmonary function have a higher exacerbation rate, lower activity rate, poor exercise tolerance and imaging evidence of initial airway disease not detectable by spirometry, as compared to nonsmokers and even asymptomatic smokers with airflow limitation [5]. In particular, this study showed that about $50 \%$ of smokers without airway obstruction have symptoms such as dyspnoea. In this category, the clinical consequences in terms of disability (e.g. reduced exercise tolerance) parallel those reported in smokers with recordable airway obstruction. The study suggests that the use of spirometry alone in dyspnoeic patients does not adequately cover the spectrum of potential (i.e. subclinical) diagnoses or symptom-related mechanisms in smokers. It also shows that it is necessary to go deeper into the physiological and/or clinical mechanisms generating dyspnoea in this large population.

In this issue of the European Respiratory Journal, ElbeHAiry et al. [6] challenge the hypothesis that adaptive mechanisms related to respiratory muscle effort and inspiratory neural drive are present in dyspnoeic current or former smokers without a spirometrically confirmed airway obstruction, i.e. those "at risk of COPD" [7]. In their small but elegant physiological study, the authors compared the trajectory of dyspnoea rating, dynamic lung mechanics and the response of respiratory muscles to the inspiratory neural drive, as obtained during a standard incremental symptom-limited cycle ergometry in smokers and healthy age-matched nonsmokers. They concluded that the increased dyspnoea perception and associated reduced exercise capacity (25\% lower maximal oxygen consumption reached in the incremental test), as

Received: June 142016 | Accepted: June 142016

Conflict of interest: Disclosures can be found alongside this article at erj.ersjournals.com

Copyright OERS 2016 
Figure 1 Exertional dyspnoea is the most important and debilitating symptom of smokers with and without airflow limitation; understanding its pathophysiology and mechanisms might allow better prevention and treatment. Image courtesy of Villa Pineta (Modena, Italy).

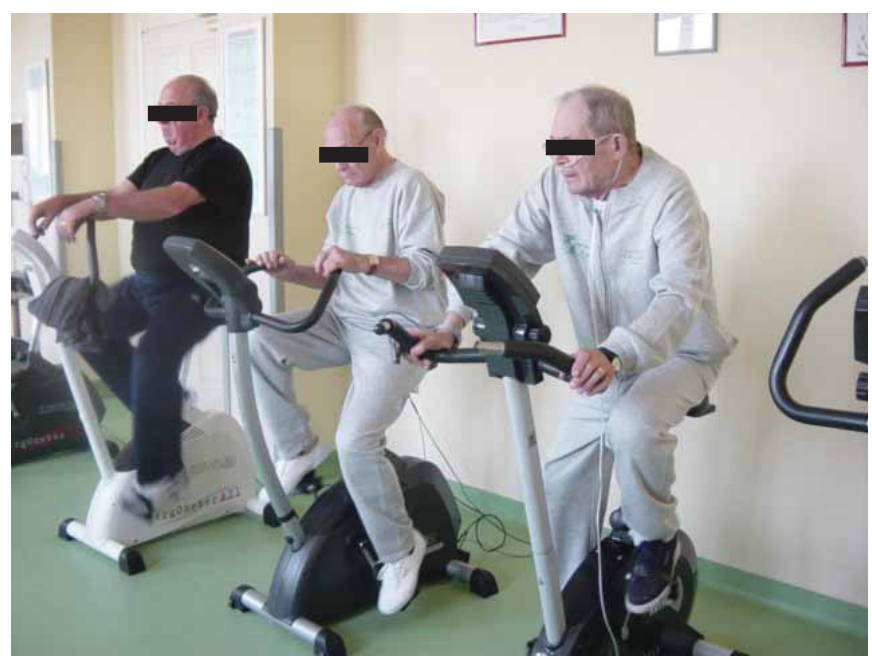

recorded in smokers who do not meet the criteria for COPD, are explained by the increased mechanical load of the respiratory system: these patients have a preserved and appropriate ventilatory response, which is not usually the case in individuals developing COPD even at a mild stage [8]. In particular, the difference in dyspnoea intensity between smokers and healthy control subjects, as assessed by the Borg scale at a comparable workload $(80 \mathrm{~W})$, is close to 2.0 Borg units, which is likely to be sensitive from a clinical point of view.

Factors contributing to the increased dyspnoea in smokers without airway obstruction have been identified as both the increased inspiratory resistive work and the increased ratio of the fractional to the maximal diaphragmatic generating pressure $\left(P \mathrm{di} / P \mathrm{di}_{\max }\right)$ and muscle activity (EMGdi/EMG $\left./ \mathrm{Edi}_{\max }\right)$, i.e. inspiratory neural drive to the diaphragm. The increased $P \mathrm{di} / P_{\mathrm{di}_{\max }}$ and $E M G d i / E M G d i_{\max }$ ratios are related to a reduction in the denominator component. This reflects the reduced voluntary contribution of the diaphragm to overall pressure generation at full lung capacity in smokers with normal spirometry, which is compatible with a greater contribution of their rib cage and accessory muscles to preserve the ventilatory response to exercise. Notwithstanding the observation of a different physiological response of the respiratory system to generate dyspnoea during maximal exercise in smokers, as discussed in the study, the causes remain unclear.

ElBEHAIRY et al. [6] hypothesise that smokers adopt a specific strategy to preserve the maximal voluntary activity of their diaphragm, which is mechanically stressed. However, it is not clear whether the diaphragm is intrinsically altered in smokers, as it is more likely to occur when COPD develops. It could be that the inflammatory process and injury from smoking alter the mechanical properties of the diaphragm. However, the EMGdi pressure level in smokers, although reduced, still falls in the range of normal [9], making this hypothesis unlikely. The authors conclude that assessing spirometry only to measure respiratory impairment related to smoking does not provide information on the true physiological mechanisms at the onset of symptoms in smokers with preserved lung volumes.

Practically, these findings extend the clinical significance of dyspnoea as a respiratory symptom that may differ because of different underlying generating mechanisms. Verbal descriptors of dyspnoea, such as heavy/fast breathing or work/effort, were found to be related to intensity level of dyspnoea in patients with COPD, particularly during exercise, and to cluster differently [10]. However, the study by ELBEHAIRY et al. [6], the objective of which was to explain the occurrence of increased dyspnoea in smokers, provides information only on alterations of the respiratory system. Indeed, the onset of dyspnoea in patients with chronic respiratory diseases is recognised as having a multifactorial, even extrapulmonary, origin [11].

Recently, the Multidimensional Dyspnoea Profile has shown that the sensory (immediate perception) and affective (emotional response) dimensions of dyspnoea can be distinguished in a large population of COPD patients [12], thus suggesting a different approach to dyspnoea. For example, a prevalent emotional response to dyspnoea may be primarily or preferably due to distractive stimuli.

The perceived intensity of dyspnoea during exercise has also been associated with the level of cortical oxygenation and cortical activation in patients with COPD [13]. Exertional dyspnoea is related to activation of the premotor cortex; the impaired cortical oxygenation, as shown in COPD patients with hypoxaemia, is thus specifically improved by an oxygen supplement. 
Finally, the frequent coexistence of extrapulmonary smoking-related conditions, such as subclinical cardiovascular diseases and metabolic abnormalities [14], may also contribute to dyspnoea, especially during an exacerbation of the disease [15]. It is known that dynamic hyperinflation in COPD patients may alter the cardiac response to exercise, which in turn affects exercise performance [16]. However, it has been shown that patients with heart failure and low resting lung diffusing capacity increase their dead space ventilation during strenuous exercise, thus leading to enhanced dyspnoea owing to inefficient ventilation-to-perfusion matching [17]. Similarly, patients with pulmonary hypertension, i.e. damage of the pulmonary vasculature, have decreased inspiratory capacity and increased dyspnoea on effort despite normal lung function [18].

It is more likely that different concomitant physiological and/or extrapulmonary mechanisms may explain the onset of dyspnoea and reduced tolerance to exercise in smokers without spirometrically confirmed COPD, because they may be related to one or more of the components of multimorbidity affecting smokers [19]. Smoking can cause or contribute to the development of various pulmonary diseases (e.g. COPD, pulmonary fibrosis, eosinophilic granuloma, tuberculosis and lung cancer) and nonrespiratory diseases (cardiovascular diseases such as hypertension, arrhythmias, ischaemic heart diseases, metabolic diseases, osteoporosis and depression), and most of these patients may present with dyspnoea as the primary symptom before diagnosis [20-22]. Thus, in smokers presenting with dyspnoea and normal spirometry, early pulmonary abnormalities such as bronchiolitis, emphysema or gas exchange abnormalities, as well as all potential chronic diseases related to smoking, should be investigated as the potential cause of dyspnoea. Clinical history, imaging, functional assessment and biomarkers [23] may be helpful.

The relevant clinical question is whether symptomatic smokers with normal lung volumes should be considered a separate clinical entity, or an early stage of COPD or other chronic disease induced by smoking [24]. Confirmation from a longitudinal study is needed. In the meantime, physiological assessment of respiratory and nonrespiratory mechanisms underlying exertional dyspnoea may produce specific categories responsive to more appropriate and targeted therapies.

\section{References}

1 Braido F, Baiardini I, Menoni S, et al. Disability in COPD and its relationship to clinical and patient-reported outcomes. Curr Med Res Opin 2011; 27: 981-986.

2 Parshall MB, Schwartzstein RM, Adams L, et al. An official American Thoracic Society statement: update on the mechanisms, assessment, and management of dyspnoea. Am J Respir Crit Care Med 2012; 185: 435-452.

3 Vitacca M, Paneroni M, Baiardi P, et al. Development of a Barthel Index based on dyspnoea for patients with respiratory diseases. In J Ch Obstr Respir Dis 2016; 11: 1199-1206.

4 Regan EA, Lynch DA, Curran-Everett D, et al. Clinical and radiologic disease in smokers with normal spirometry. JAMA Intern Med 2015; 175: 1539-1549.

5 Woodruff PG, Barr RG, Bleecker E, et al. Clinical significance of symptoms in smokers with preserved pulmonary function. N Engl J Med 2016; 11: 41-58.

6 Elbehairy AF, Guenette JA, Faisal A, et al. Mechanisms of exertional dyspnoea in symptomatic smokers without COPD. Eur Respir J 2016; 48: 694-705.

7 Global Initiative for Chronic Obstructive Lung Disease. Global Strategy for the Diagnosis, Management and Prevention of COPD. 2015. Available from: www.goldcopd.org

8 Guenette JA, Chin RC, Cheng S, et al. Mechanisms of exercise intolerance in global initiative for chronic obstructive lung disease grade 1 COPD. Eur Respir J 2014; 44: 1177-1187.

9 De Troyer A, Estenne M. Limitations of measurement of transdiaphragmatic pressure in detecting diaphragmatic weakness. Thorax 1981; 36: 169-174.

10 von Leupoldt A, Balewski S, Petersen S, et al. Verbal descriptors of dyspnoea in patients with COPD at different intensity levels of dyspnoea. Chest 2007; 132: 141-147.

11 Laviolette L, Laveneziana P. Dyspnoea: a multidimensional and multidisciplinary approach. Eur Respir J 2014; 43: $1750-1762$.

12 Morélot-Panzini C, Gilet H, Aguilaniu B, et al. Real-life assessment of the multidimensional nature of dyspnoea in COPD outpatients. Eur Respir J 2016; 47: 1668-1679.

13 Higashimoto Y, Honda N, Yamagata T, et al. Exertional dyspnoea and cortical oxygenation in patients with COPD. Eur Respir J 2015; 46: 1615-1624.

14 Lopez-Campos JL, Agustí A. Heterogeneity of chronic obstructive pulmonary disease exacerbations: a two-axes classification proposal. Lancet Respir Med 2015; 3: 729-734.

15 Beghé B, Verduri A, Roca M, et al. Exacerbation of respiratory symptoms in COPD patients may not be exacerbations of COPD. Eur Respir J 2013; 41: 993-995.

16 Tzani P, Aiello M, Elia D, et al. Dynamic hyperinflation is associated with a poor cardiovascular response to exercise in COPD patients. Respir Res 2011; 12: 150.

17 Kee K, Stuart-Andrews C, Ellis MJ, et al. Increased dead space ventilation mediates reduced exercise capacity in systolic heart failure. Am J Respir Crit Care Med 2016; 193: 1292-1300.

18 Laveneziana $\mathrm{P}$, Humbert $\mathrm{M}$, Godinas $\mathrm{L}$, et al. Inspiratory muscle function, dynamic hyperinflation and exertional dyspnoea in pulmonary arterial hypertension. Eur Respir J 2015; 45: 1495-1498.

19 National Clinical Guidelines Center. Multimorbidity: clinical assessment and management, NICE guidelines 2016. www.nice.org.uk/guidance/indevelopment/gid-cgwave0704 Date last accessed: June 7, 2016.

20 US Department of Health and Human Services. The Health Consequences of Smoking: 50 Years of Progress. A Report of the Surgeon General. Atlanta, US Department of Health and Human Services, 2014. 
21 Carter BD, Abnet CC, Feskanich D, et al. Smoking and mortality - beyond established causes. N Engl J Med 2015; 372: 631-640.

22 Hayen A, Herigstad M, Pattinson KT. Understanding dyspnoea as a complex individual experience. Maturitas 2013; 76: 45-50.

23 Suzuki T, Lyon A, Saggar R, et al. Biomarkers of acute cardiovascular and pulmonary diseases. Eur Heart J Acute Cardiovasc Care 2016 [in press DOI: 10.1177/2048872616652309].

24 Fabbri LM. Smoking, not COPD, as the disease. N Engl J Med 2016; 374: 1885-1886. 